\title{
Permanence and Global Attractivity of a Discrete Logistic Model with Impulses
}

\author{
Chunyu Gao ${ }^{1,2}$ and Qingxi Guo ${ }^{1}$ \\ ${ }^{1}$ Center for Ecological Research, Northeast Forestry University, Harbin 150040, China \\ ${ }^{2}$ Department of Mathematics, Northeast Agricultural University, Harbin 150030, China \\ Correspondence should be addressed to Qingxi Guo; guoqingxi_2000@163.com
}

Received 20 April 2013; Accepted 3 August 2013

Academic Editor: Maoan Han

Copyright (c) 2013 C. Gao and Q. Guo. This is an open access article distributed under the Creative Commons Attribution License, which permits unrestricted use, distribution, and reproduction in any medium, provided the original work is properly cited.

By piecewise Euler method, we construct a discrete logistic equation with impulses. The constructed model is more easily implemented at computer and is a better analogue of the continuous-time dynamic system. The dynamic behaviors of the constructed model are investigated. Sufficient conditions which guarantee the permanence and the global attractivity of positive solutions of the model are obtained. Numerical simulations show the feasibility of the main results.

\section{Introduction}

It is well known that impulsive differential equations have been considered by many authors (see, e.g., [1-7]). Such equations may exhibit several real world phenomena, such as rhythmical beating, merging of solutions, and noncontinuity of solutions. Since it is much richer than the corresponding theory of differential equations without impulsive effects, the theory of impulsive differential equations is emerging as an important area of investigation of model.

At all times, continuous-time dynamic systems play an important role in control theory, networks design, and so on. However, with the development of computer techniques, when implementing the continuous-time dynamic systems for computer simulation, for experimental or computational purposes, it is essential to formulate discrete-time dynamic systems which are an analogue of the continuoustime dynamic systems. These discrete-time systems, which are described by difference equations, inherit the similar dynamical characteristics. Because of that, more and more researchers pay their attention to the dynamical behaviors of difference equations (see [8-12]). However, few papers investigate the discrete model with impulses (see [13-17]). The main difficulty of constructing discrete model with impulses is how to describe impulsive moment. The main purpose of this paper is to construct the discrete model with impulses and investigate the permanence and global stability of the discrete model with impulses.

By piecewise Euler method, we construct the following discrete logistic model with impulses:

$$
\begin{gathered}
x_{m_{k}+l+1}=x_{m_{k}+l} \exp \left\{r\left(1-\frac{x_{m_{k}+l}}{K}\right)\right\}, \\
x_{m_{k}+0}=\left(1+b_{k}\right) x_{m_{k}}, \\
l=0,1,2, \ldots,\left(m_{k+1}-m_{k}-2\right),\left(m_{k+1}-m_{k}-1\right), \\
k=0,1,2, \ldots,
\end{gathered}
$$

where the fixed moments of time $m_{k}$ satisfy $m_{0}=0, m_{k}<$ $m_{k+1}$, and $\lim _{k \rightarrow \infty} m_{k}=+\infty . r$ is the intrinsic rate and $K$ is the carrying capacity of the system; $x_{0}=x_{0+0}$ is the initial value.

In this paper, we always assume that $0<B_{1}<1+b_{k}<B_{2}$, $k=1,2,3, \ldots$.

We simply explain model (1).

It is obvious that $x_{m_{k}+l}>0, k=0,1,2, \ldots, l=0,1,2, \ldots$, $\left(m_{k+1}-m_{k}-2\right),\left(m_{k+1}-m_{k}-1\right)$, for $x_{0}>0$.

When $k=0, x_{l+1}=x_{l} \exp \left\{r\left(1-\left(x_{l} / K\right)\right)\right\}, l=0,1$, $2, \ldots, m_{1}-1$. We can obtain that $x_{1}, x_{2}, \ldots, x_{m_{1}}$ and $x_{m_{1}+0}=$ $\left(1+b_{1}\right) x_{m_{1}}$. 
When $k=1, x_{m_{1}+l+1}=x_{m_{1}+l} \exp \left\{r\left(1-\left(x_{m_{1}+l} / K\right)\right)\right\}, l=$ $0,1,2, \ldots, m_{2}-m_{1}-1$. We can obtain that $x_{m_{1}+1}, x_{m_{1}+2}$, $\ldots, x_{m_{2}}$, and $x_{m_{2}+0}=\left(1+b_{2}\right) x_{m_{2}}$, and so on.

In some papers, authors use $m_{k}^{+}$to denote impulsive moment (see [15]). It is obvious that describing the impulsive moment of model (1) is easily realized at computer. In addition, some authors use $m_{k}+1$ to denote impulsive moment $($ see $[13,16])$. Compared with it, model $(1)$ is a better analogue of the continuous-time dynamic system.

The organization of this paper is as follows. In the next two sections, we give sufficient conditions on permanence and global stability of system (1), respectively. In Section 4, two examples are given. To conclude this paper, a discussion follows in Section 5.

\section{Permanence}

For convenience, let $n=m_{k}+l$.

Definition 1. The species $x_{n}$ of system (1) is said to be permanence if there exist positive constants $M$ and $m$ such that each positive solution $x_{n}$ of the system satisfies

$$
m \leq \liminf _{n \rightarrow \infty} x_{n} \leq \limsup _{n \rightarrow \infty} x_{n} \leq M .
$$

If the species of the system is permanence, then the system is called permanent.

Lemma 2. There exists a positive constant $M$, such that for every solution $x_{n}$ of system (1), one has

$$
x_{n} \leq M, \text { for } n>0 .
$$

Proof. To prove (3), we have two cases.

Case $I$. For any $x_{0}>0$, we have

$$
\begin{aligned}
x_{n+1} & =x_{n} \exp \left\{r\left(1-\frac{x_{n}}{K}\right)\right\} \\
& \leq \frac{K \exp \{r-1\}}{r} \triangleq M_{1} .
\end{aligned}
$$

Here, we used

$$
\max _{x \geq 0}\{x \exp \{b-a x\}\}=\frac{\exp \{b-1\}}{a} .
$$

Case II. $n=m_{k}+0, k=1,2,3, \ldots$. We have

$$
\begin{aligned}
x_{m_{k}+0} & =\left(1+b_{k}\right) x_{m_{k}} \\
& =\left(1+b_{k}\right) x_{m_{k}-1} \exp \left\{r\left(1-\frac{x_{m_{k}-1}}{K}\right)\right\} \\
& \leq B_{2} \frac{K \exp \{r-1\}}{r} \triangleq \widehat{M}_{1} .
\end{aligned}
$$

Let $M=\max \left\{M_{1}, \widehat{M}_{1}\right\}$. This completes the proof.

Lemma 3. Assume there exists a positive constant $B_{3}$, such that $B_{3}<\prod_{k}^{\infty}\left(1+b_{k}\right)$. Then there exist two positive constants $m$ and $n_{1}$, such that for every solution $x_{n}$ of system (1), one has $x_{n}>m$ for $n>n_{1}$.
Proof

Step I. Let $0<m^{\prime}<K$. We will firstly prove that there exists $n_{1}$ such that $x_{n_{1}} \geq m^{\prime}$. Otherwise, for all $n, x_{n}<m^{\prime}$,

$$
\begin{aligned}
x_{n+1} & =x_{n} \exp \left\{r\left(1-\frac{x_{n}}{K}\right)\right\} \\
& \geq x_{n} \exp \left\{r\left(1-\frac{m^{\prime}}{K}\right)\right\} .
\end{aligned}
$$

Therefore, there exists a constant $k^{\prime}$, such that if $k>k^{\prime}$,

$$
\begin{aligned}
x_{m_{k+1}} & \geq x_{m_{k+1}-1} \exp \left\{r\left(1-\frac{m^{\prime}}{K}\right)\right\} \\
& \geq x_{m_{k+1}-2} \exp \left\{2 r\left(1-\frac{m^{\prime}}{K}\right)\right\} \\
& \vdots \\
& \geq x_{m_{k}+0} \exp \left\{\left(m_{k+1}-m_{k}\right) r\left(1-\frac{m^{\prime}}{K}\right)\right\} \\
& =\left(1+b_{k}\right) x_{m_{k}} \exp \left\{\left(m_{k+1}-m_{k}\right) r\left(1-\frac{m^{\prime}}{K}\right)\right\} \\
& \geq x_{m_{k^{\prime}}} \prod_{i=k^{\prime}}^{k}\left(1+b_{i}\right) \exp \left\{\left(m_{k+1}-m_{k^{\prime}}\right) r\left(1-\frac{m^{\prime}}{K}\right)\right\} \\
& \longrightarrow \infty
\end{aligned}
$$

as $k \rightarrow \infty$, which contradicts the boundedness of $x_{n}$. Hence, we conclude that there exists $n_{1}$ such that $x_{n_{1}} \geq m^{\prime}$.

Step II. If $x_{n} \geq m^{\prime}$, for all $n \geq n_{1}$, then our aim is achieved. Otherwise, $x_{n}<m^{\prime}$ for some $n>n_{1}$. Set $n^{*}=\min _{n>n_{1}}\left\{x_{n}<\right.$ $\left.m^{\prime}\right\}$. We have $x_{n} \geq m^{\prime}$ for $n \in\left[n_{1}, n^{*}\right)$.

Assume that $n^{*} \in\left[m_{k}, m_{k+1}\right)$. It is easy to see that $x_{n^{*}}<$ $m^{\prime}, x_{n^{*}-1} \geq m^{\prime}$.

There are two possible cases for $n \in\left[n^{*}, m_{k+1}\right)$.

Case A. There exists a $n^{\prime \prime} \in\left[n^{*}, m_{k+1}\right)$, such that $x_{n^{\prime \prime}} \geq m^{\prime}$. Let $\widehat{n}=\min _{n>n^{*}}\left\{x_{n} \geq m^{\prime}\right\}$; then $x_{n}<m^{\prime}$ for $n \in\left[n^{*}, \widehat{n}\right)$ and $x_{\widehat{n}} \geq m^{\prime}$. Select an $M$ such that

$$
x_{n}<M, \quad \max \left(1, B_{1}\right) \exp \left(r\left(1-\frac{M}{K}\right)\right)<1 .
$$

If $n^{*} \neq m_{k}+0$, then

$$
\begin{aligned}
x_{n^{*}} & =x_{n^{*}-1} \exp \left(r\left(1-\frac{x_{n^{*}-1}}{K}\right)\right) \\
& \geq m^{\prime} \exp \left(r\left(1-\frac{M}{K}\right)\right) .
\end{aligned}
$$

If $n^{*}=m_{k}+0$, then $m_{k} \geq n_{1}$.

Case $1\left(m_{k}=n_{1}\right)$. Consider

$$
x_{n^{*}}=\left(1+b_{k}\right) x_{m_{k}} \geq B_{1} m^{\prime} .
$$


Case $2\left(m_{k}>n_{1}\right)$. Consider

$$
\begin{aligned}
x_{n^{*}} & =\left(1+b_{k}\right) x_{m_{k}} \\
& \geq\left(1+b_{k}\right) x_{m_{k}-1} \exp \left(r\left(1-\frac{x_{m_{k}-1}}{K}\right)\right) \\
& \geq\left(1+b_{k}\right) m^{\prime} \exp \left(r\left(1-\frac{M}{K}\right)\right) \\
& \geq B_{1} m^{\prime} \exp \left(r\left(1-\frac{M}{K}\right)\right) .
\end{aligned}
$$

For $n \in\left[n^{*}, \widehat{n}-1\right)$,

$$
\begin{aligned}
x_{n+1} & \geq x_{n} \exp \left(r\left(1-\frac{m^{\prime}}{K}\right)\right) \\
& \vdots \\
& \geq x_{n^{*}} \exp \left(\left(n+1-n^{*}\right) r\left(1-\frac{m^{\prime}}{K}\right)\right) .
\end{aligned}
$$

If $n^{*} \neq m_{k}+0$, then

$$
\begin{aligned}
x_{n+1} & \geq x_{n^{*}} \exp \left(\left(n+1-n^{*}\right) r\left(1-\frac{m^{\prime}}{K}\right)\right) \\
& \geq m^{\prime} \exp \left(r\left(1-\frac{M}{K}\right)\right) \exp \left(r\left(1-\frac{m^{\prime}}{K}\right)\right) \\
& \geq m^{\prime} \exp \left(r\left(1-\frac{M}{K}\right)\right) \triangleq \bar{m}
\end{aligned}
$$

If $n^{*}=m_{k}+0$, then

$$
\begin{aligned}
x_{n+1} \geq & x_{n^{*}} \exp \left(\left(n+1-n^{*}\right) r\left(1-\frac{m^{\prime}}{K}\right)\right) \\
\geq & \left(1+b_{k}\right) m^{\prime} \exp \left(r\left(1-\frac{M}{K}\right)\right) \\
& \times \exp \left(\left(n+1-n^{*}\right) r\left(1-\frac{m^{\prime}}{K}\right)\right) \\
\geq & \left(1+b_{k}\right) m^{\prime} \exp \left(r\left(1-\frac{M}{K}\right)\right) \exp \left(r\left(1-\frac{m^{\prime}}{K}\right)\right) \\
\geq & B_{1} m^{\prime} \exp \left(r\left(1-\frac{M}{K}\right)\right) \triangleq \widetilde{m} .
\end{aligned}
$$

The argument of Step II can be continued since $x_{\widehat{n}} \geq m^{\prime}$, and we have $x_{n} \geq m=\min (\widetilde{m}, \bar{m})$ for all $n>n_{1}$.

Case B. $x_{n}<m^{\prime}$ for $n \in\left[n^{*}, m_{k+1}\right)$. If $x_{n} \geq m^{\prime}$ for all $n \geq$ $m_{k+1}$, then our aim is achieved. Otherwise, $x_{n}<m^{\prime}$ for some $n>m_{k+1}$. Set $\bar{n}=\min _{n>m_{k+1}}\left\{x_{n}<m^{\prime}\right\}$.

For $n>\bar{n}$, the argument of step II can be continued since $x_{n} \geq m^{\prime}$ for $n \in\left[m_{k+1}, \bar{n}\right)$; hence $x_{n} \geq m=\min (\widetilde{m}, \bar{m})$, for all $n>m_{k+1}$. The proof is complete.
Combining Lemmas 2 and 3, we have proved the main result of this paper, which is stated next.

Theorem 4. If the condition of Lemma 3 holds, system (1) is permanent.

\section{Global Attractivity of the Positive Solution}

Next we discuss the global attractivity of the positive solution of system (1). In the following we say a positive solution of system (1) is globally asymptotically stable if it attracts all other positive solutions of the system. form:

Let $\widetilde{x_{n}}=\ln \left(x_{n}\right)$. Then system (1) becomes the following

$$
\begin{aligned}
& \widetilde{x_{n+1}}=\widetilde{x_{n}}+r\left(1-\frac{e^{\widetilde{x_{n}}}}{K}\right), \\
& \widetilde{x_{m_{k}+0}}=\ln \left(1+b_{k}\right)+\widetilde{x_{m_{k}}} .
\end{aligned}
$$

Theorem 5. If $0<M<2(K / r)$, then for any two positive solutions $x_{n}$ and $y_{n}$ of system (1), one has

$$
\lim _{n \rightarrow \infty}\left|x_{n}-y_{n}\right|=0
$$

Proof. Let $\widetilde{x_{n}}=\ln \left(x_{n}\right)$ and let $\widetilde{y_{n}}=\ln \left(y_{n}\right)$. Then

$$
\begin{aligned}
\left|\widetilde{x_{n+1}}-\widetilde{y_{n+1}}\right| & =\left|\widetilde{x_{n}}-\widetilde{y_{n}}-\frac{r}{K}\left(e^{\widetilde{x_{n}}}-e^{\widetilde{y_{n}}}\right)\right| \\
& =\left|\widetilde{x_{n}}-\widetilde{y_{n}}-\frac{r}{K} e^{\xi(n)}\left(\widetilde{x_{n}}-\widetilde{y_{n}}\right)\right| \\
& =\left|\left(1-\frac{r}{K} e^{\xi(n)}\right)\right|\left|\left(\widetilde{x_{n}}-\widetilde{y_{n}}\right)\right|,
\end{aligned}
$$

where $e^{\xi(n)}$ is between $x_{n}$ and $y_{n}$ and $0<m<e^{\xi(n)}<M$ for sufficiently large $n$.

In view of $0<M<2(K / r)$ such that

$$
\left|\left(1-\frac{r}{K} e^{\xi(n)}\right)\right|<1
$$

hence,

$$
\lim _{n \rightarrow \infty}\left|\widetilde{x_{n}}-\widetilde{y_{n}}\right|=0
$$

which implies that

$$
\lim _{n \rightarrow \infty}\left|x_{n}-y_{n}\right|=0
$$

This completes the proof.

\section{Examples and Numerical Simulation}

Example 1. Corresponding to the system (1), we assume that

$$
\begin{gathered}
r=0.2, \quad K=100, \quad m_{0}=0, \\
m_{k}=2^{k}, \quad b_{k}=\sin k, \quad k=1,2, \ldots .
\end{gathered}
$$

It is easy to see that the conditions in Theorem 4 are verified. Therefore, (1) is permanent (see Figure 1). 


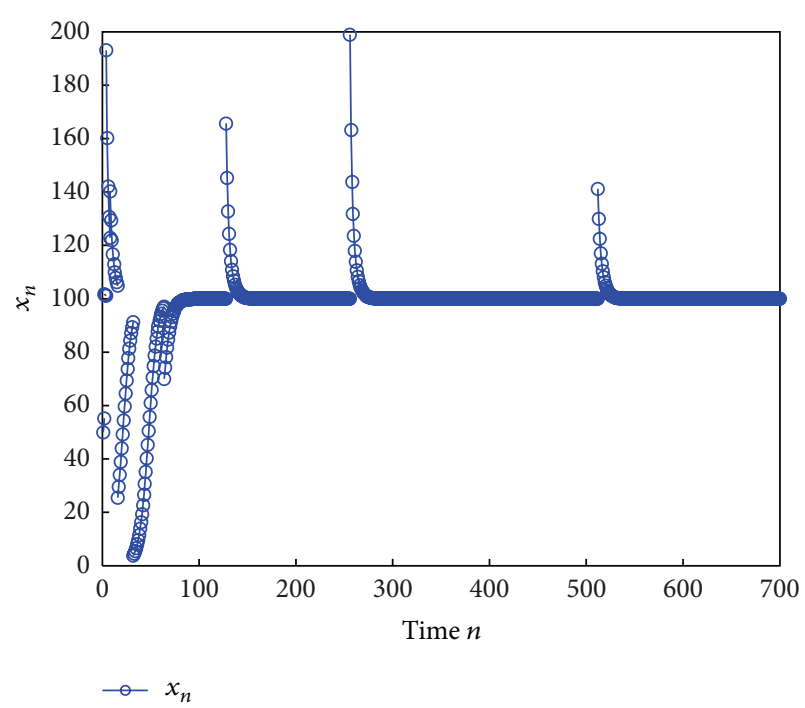

Figure 1: Permanence of system (1) with initial condition $x_{0}=50$.

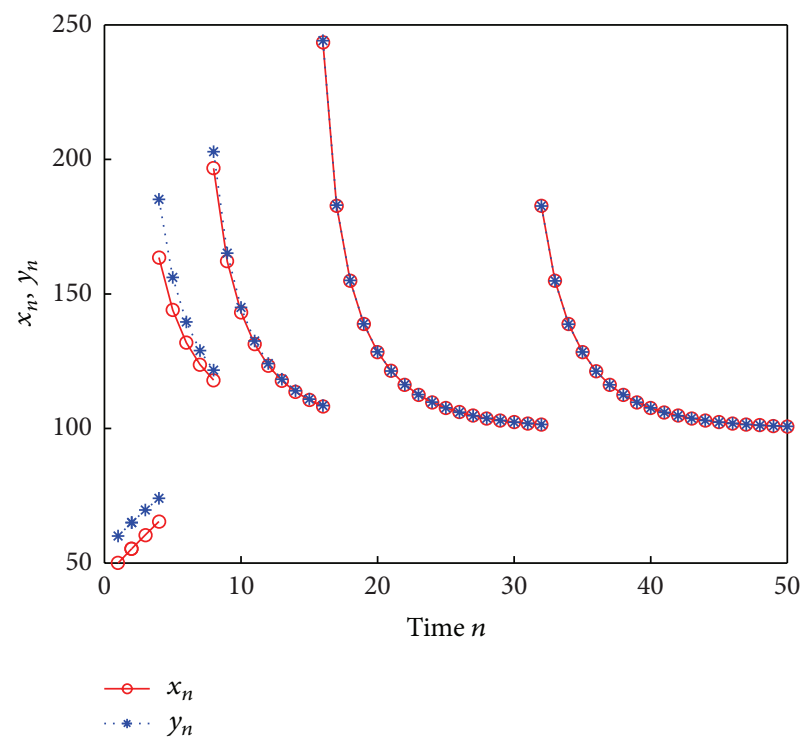

FIGURE 2: Global attractivity of system (1) with initial conditions $x_{0}=50$ and $y_{0}=60$.

Example 2. Corresponding to the system (1), we assume that

$$
\begin{gathered}
r=0.2, \quad K=100, \quad m_{0}=0, \\
m_{k}=2^{k}, \quad b_{k}=\left(1+(-1)^{k} \frac{1}{k}\right), \quad k=1,2, \ldots .
\end{gathered}
$$

It is easy to see that the conditions in Theorem 5 are verified. Therefore, (1) is global attractivity. Our numerical simulation supports our result (see Figure 2).

\section{Conclusion}

In this paper, by piecewise Euler method, we construct a discrete logistic equation with impulses. The model gives a new form of describing the impulsive moment, and the model is more easily implemented at computer and is a better analogue of the continuous-time dynamic system. The permanence and the global attractivity of positive solutions of the model are investigated. In our opinion, this discrete idea of the paper can apply to more complex model with impulses, such as model with impulses and delay and multigroup mode with impulses and delay. The other dynamic behaviors of discrete model can be researched, such as stability, periodic solution, and invariant sets.

\section{Acknowledgment}

This work is supported by the Key Projects in the National Science \& Technology Pillar Program (no. 2011BAD37B01).

\section{References}

[1] J. K. Hale, Theory of Functional Differential Equations, Springer, New York, NY, USA, 1977.

[2] V. Lakshmikantham, D. D. Baĭnov, and P. S. Simeonov, Theory of Impulsive Differential Equations, World Scientific, Singapore, 1989.

[3] K. Gopalsamy and B. G. Zhang, "On delay differential equations with impulses," Journal of Mathematical Analysis and Applications, vol. 139, no. 1, pp. 110-122, 1989.

[4] D. Y. Xu and Z. C. Yang, "Impulsive delay differential inequality and stability of neural networks," Journal of Mathematical Analysis and Applications, vol. 305, no. 1, pp. 107-120, 2005.

[5] V. Lakshmikantham, X. Liu, and S. Sathananthan, "Impulsive integro-differential equations and extension of Lyapunov's method," Applicable Analysis, vol. 32, no. 3-4, pp. 203-214, 1989.

[6] A. Zhao and J. Yan, "Asymptotic behavior of solutions of impulsive delay differential equations," Journal of Mathematical Analysis and Applications, vol. 201, no. 3, pp. 943-954, 1996.

[7] Y. P. Xing and M. A. Han, "A new approach to stability of impulsive functional differential equations," Applied Mathematics and Computation, vol. 151, no. 3, pp. 835-847, 2004.

[8] M. S. Peng, "Oscillation theorems of second-order nonlinear neutral delay difference equations with impulses," Computers \& Mathematics with Applications, vol. 44, no. 5-6, pp. 741-748, 2002.

[9] Q. Q. Zhang, "On a linear delay difference equation with impulses," Annals of Differential Equations, vol. 18, no. 2, pp. 197204, 2002.

[10] Z. M. He and X. M. Zhang, "Monotone iterative technique for first order impulsive difference equations with periodic boundary conditions," Applied Mathematics and Computation, vol. 156, no. 3, pp. 605-620, 2004.

[11] R. Z. Abdullin, "Stability of nonlinear difference equations with pulse actions: a comparison method," Automation and Remote Control, vol. 61, no. 11, pp. 1796-1807, 2000.

[12] R. Z. Abdullin, "Stability of difference equations with impulse actions at moments of time that depend on the vector state," Automation and Remote Control, vol. 58, no. 7, pp. 1092-1100, 1997.

[13] B. Liu and D. J. Hill, "Uniform stability and ISS of discrete-time impulsive hybrid systems," Nonlinear Analysis. Hybrid Systems, vol. 4, no. 2, pp. 319-333, 2010. 
[14] H. Xu, Y. Chen, and K. L. Teo, "Global exponential stability of impulsive discrete-time neural networks with time-varying delays," Applied Mathematics and Computation, vol. 217, no. 2, pp. 537-544, 2010.

[15] Z. Zhang and X. Liu, "Robust stability of uncertain discrete impulsive switching systems," Computers \& Mathematics with Applications, vol. 58, no. 2, pp. 380-389, 2009.

[16] S. Wu, C. Li, X. Liao, and S. Duan, "Exponential stability of impulsive discrete systems with time delay and applications in stochastic neural networks: a Razumikhin approach," Neurocomputing, vol. 82, pp. 29-36, 2012.

[17] H. Liang, M. Liu, and M. Song, "Extinction and permanence of the numerical solution of a two-prey one-predator system with impulsive effect," International Journal of Computer Mathematics, vol. 88, no. 6, pp. 1305-1325, 2011. 


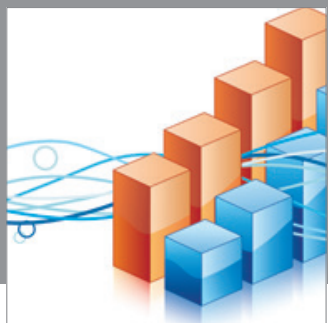

Advances in

Operations Research

mansans

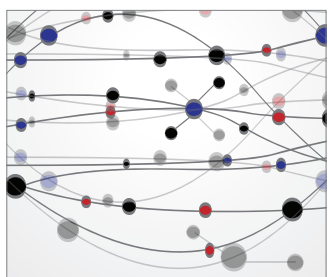

The Scientific World Journal
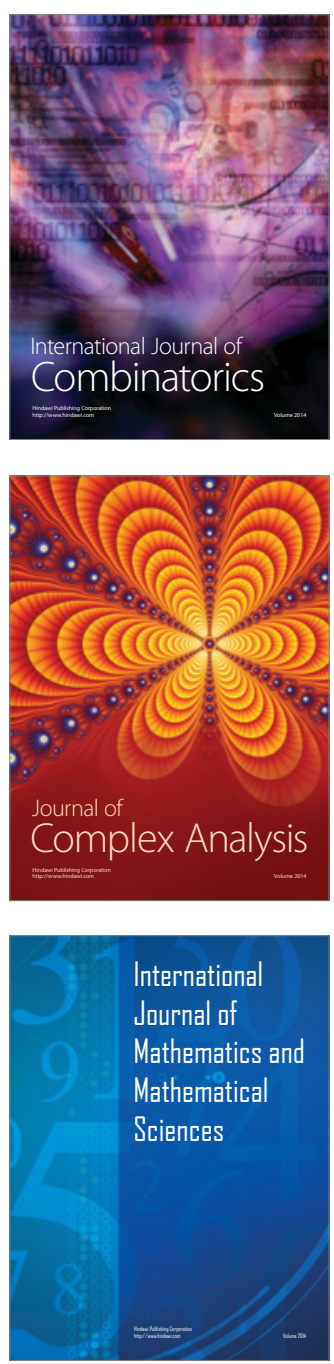
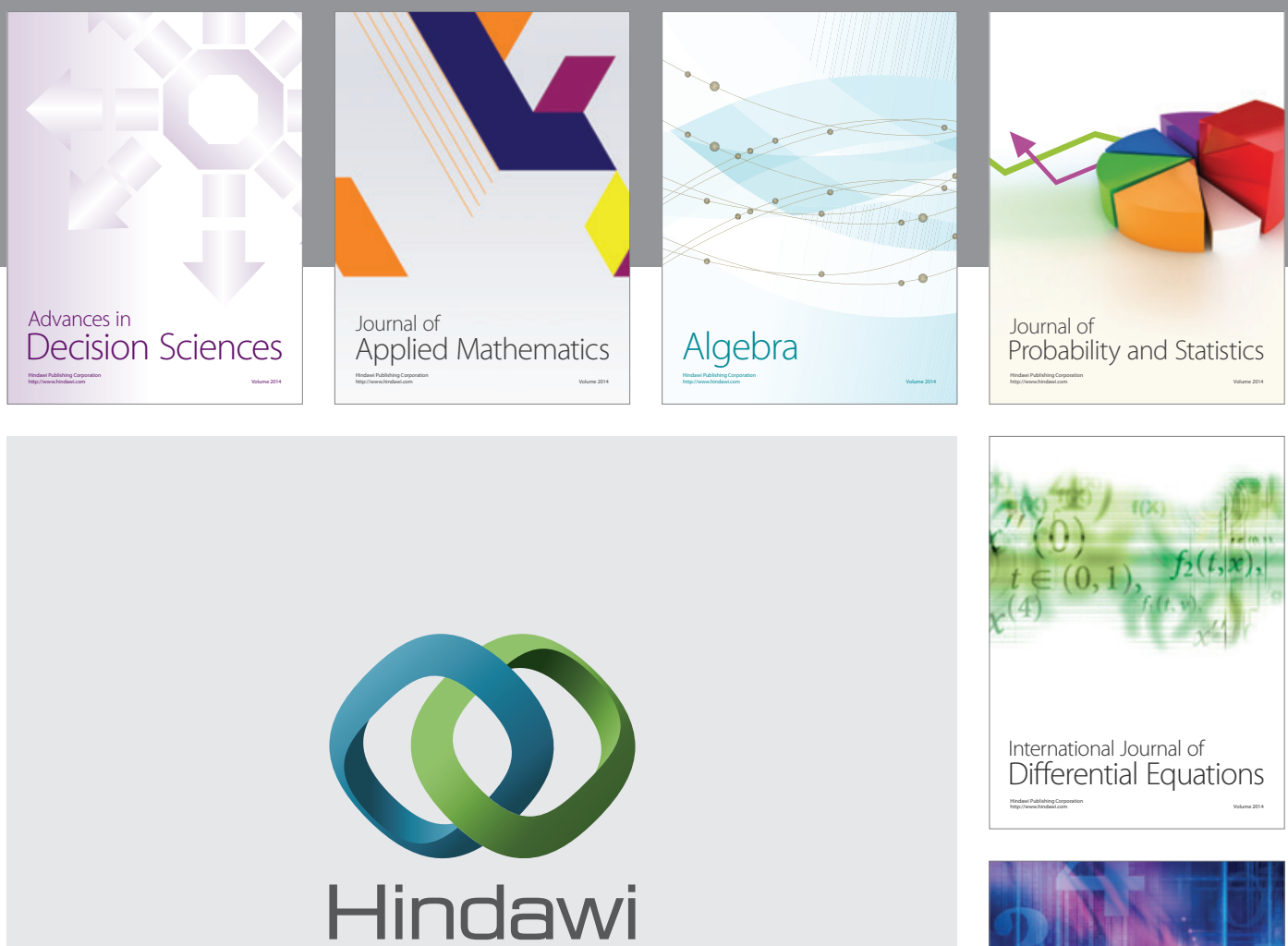

Submit your manuscripts at http://www.hindawi.com
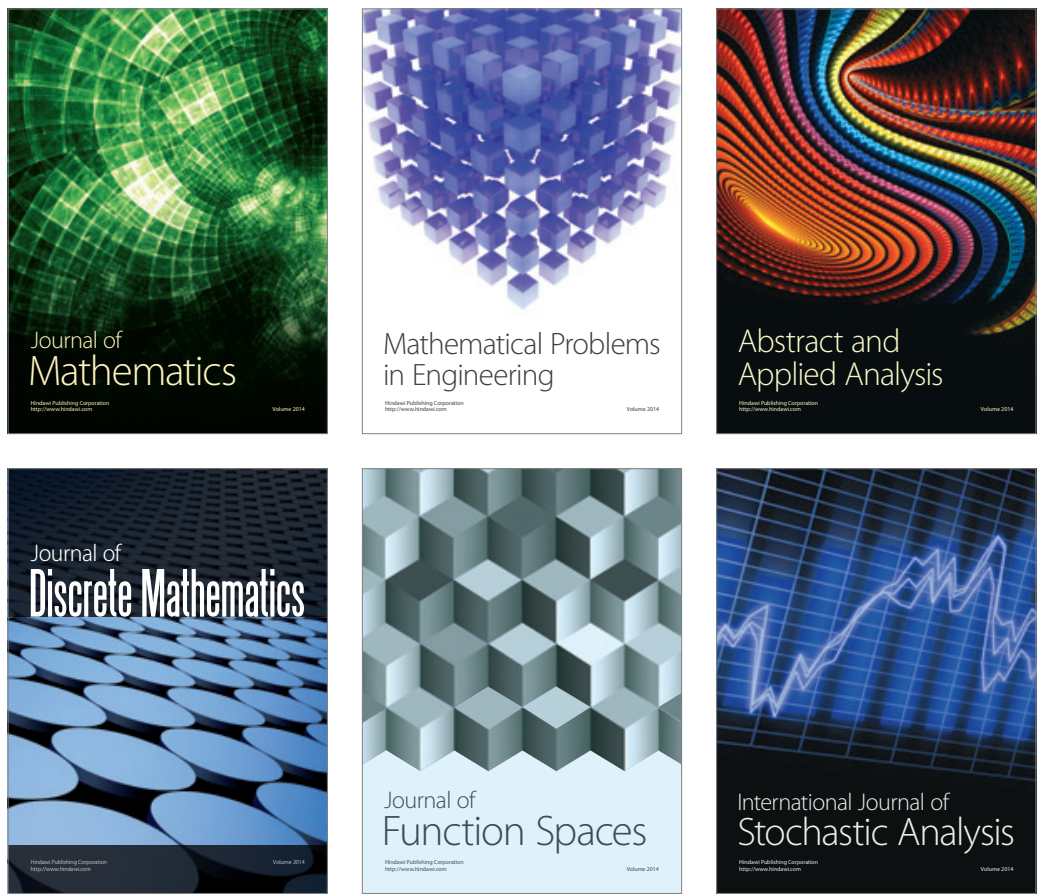

Journal of

Function Spaces

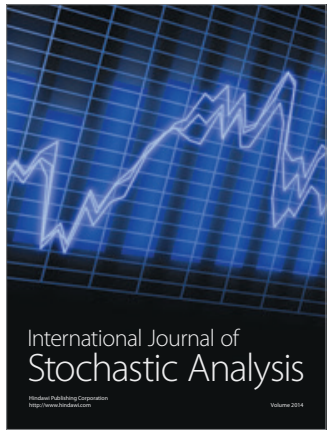

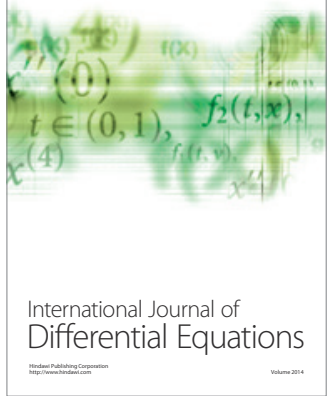
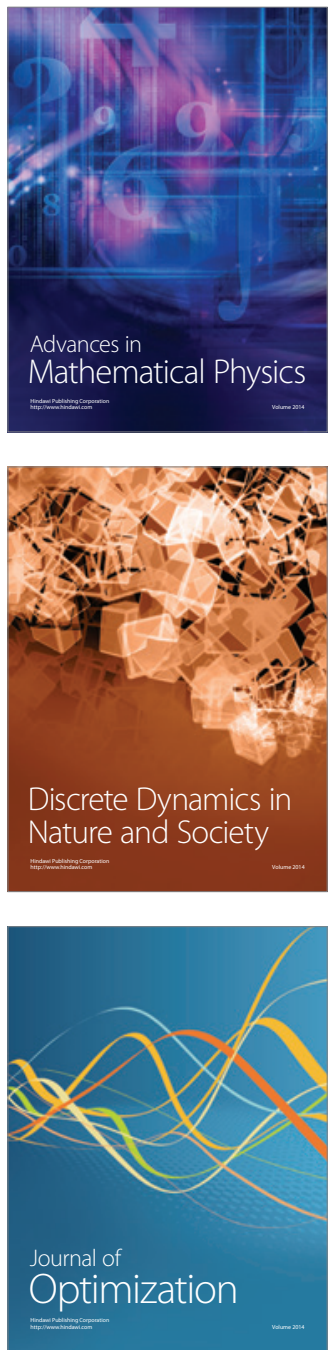